\title{
Applying scheduling and tuning to on-line parallel tomography
}

\author{
Shava Smallen ${ }^{\mathrm{a}}$, Henri Casanova ${ }^{\mathrm{b}, \mathrm{c}}$ and Francine Berman ${ }^{\mathrm{b}, \mathrm{c}}$ \\ ${ }^{a}$ Computer Science Department, Indiana University, Bloomington, IN 47404-7104, USA \\ Tel.: +1812855 4081; Fax: +18128554829; E-mail: ssmallen@cs.indiana.edu \\ ${ }^{\mathrm{b}}$ Computer Science and Engineering Department, University of California, San Diego, 9500 Gilman Dr, La Jolla, \\ CA 92093-0114, USA \\ E-mail: \{casanova,berman\}@cs.ucsd.edu \\ 'San Diego Supercomputer Center, 9500 Gilman Dr, La Jolla, CA 92093-0505, USA
}

\begin{abstract}
Tomography is a popular technique to reconstruct the three-dimensional structure of an object from a series of twodimensional projections. Tomography is resource-intensive and deployment of a parallel implementation onto Computational Grid platforms has been studied in previous work. In this work, we address on-line execution of the application where computation is performed as data is collected from an on-line instrument. The goal is to compute incremental 3-D reconstructions that provide quasi-real-time feedback to the user.

We model on-line parallel tomography as a tunable application: trade-offs between resolution of the reconstruction and frequency of feedback can be used to accommodate various resource availabilities. We demonstrate that application scheduling/tuning can be framed as multiple constrained optimization problems and evaluate our methodology in simulation. Our results show that prediction of dynamic network performance is key to efficient scheduling and that tunability allows for production runs of on-line parallel tomography in Computational Grid environments.
\end{abstract}

\section{Introduction}

Tomography is a widely used technique to reconstruct the three-dimensional structure of an object from a series of two-dimensional projections [1]. Furthermore, several tomography algorithms are inherently parallel [1-3]. With these algorithms, the threedimensional volume or tomogram can be decomposed into slices such that each slice is computed independently.

One can envision two scenarios for running parallel tomography: off-line and on-line. In off-line parallel tomography, the user runs tomography on a dataset that resides on secondary storage to obtain a single, high-resolution tomogram as quickly as possible. Conversely, on-line parallel tomography operates on data as it is collected from an on-line instrument. The goal is to compute successive tomograms in quasi-real-time to provide feedback on the quality of the data acquisition.
In previous work, we and our collaborators developed a parallel implementation of off-line tomography called GTOMO (Grid TOMOgraphy) [4]. This implementation is currently used in production at the $\mathrm{Na}$ tional Center for Microscopy and Imaging Research (NCMIR). GTOMO is used to reconstruct the threedimensional structure of biological specimens at the cellular and sub-cellular level using two-dimensional projections collected from a powerful electron microscope.

In this paper, we address the on-line scenario. We target on-line parallel tomography to a Computational Grid (or Grid for short) [5,6] composed of multi-user workstation clusters and supercomputers. Executing applications in this type of computing environment can be challenging because resource availability is dynamic. Our results show that quasi-real-time execution of on-line parallel tomography can be achieved using a strategy that combines application tunability [7] with application-level scheduling [8,9]. This strategy 
has been incorporated into GTOMO and will be put in production at NCMIR.

This paper is organized as follows. In Section 2, we briefly review our work on off-line parallel tomography and describe application-level scheduling. We also describe on-line parallel tomography and motivate its implementation as a tunable application. Section 3 details our scheduling strategy for deploying on-line parallel tomography on a Grid. Simulation results are given in Section 4. Section 5 discusses related work and Section 6 concludes the paper.

\section{From off-line to on-line}

\subsection{Tomography at NCMIR}

During a tomography experiment at NCMIR, a specimen is placed under an electron microscope and rotated about a single axis while $p$ projections are acquired using a charge-coupled device (CCD) camera. Typically 61 projections are acquired. The size of each projection depends on the resolution of the CCD camera, currently either $1 k \times 1 k$ or $2 k \times 2 k$.

We define a tomography experiment as $E=$ $(p, x, y, z)$ where $p$ is the number of projections, $x$ and $y$ are the dimensions of the projections and $z$ is the thickness of the object; $x, y$, and $z$ are measured in pixels. Given the resolution of the CCD cameras, $(61,1024,1024,300)$ and $(61,2048,2048,600)$ are representative examples of NCMIR experiments.

The tomography reconstruction techniques used by NCMIR (R-weighted backprojection [10], ART [11], SIRT [12]) are embarrassingly parallel. Figure 1 illustrates the parallelism of these techniques. The information required to produce the $i^{t h} \mathrm{X}-\mathrm{Z}$ slice of the threedimensional volume (or tomogram) is the $i^{\text {th }}$ scanline from all projections. Therefore, the three-dimensional volume can be decomposed into a series of $\mathrm{X}-\mathrm{Z}$ slices where each slice is computed independently of the others.

\subsection{Off-line parallel tomography}

The target computing platform for NCMIR is a Grid composed of multi-user workstation clusters and supercomputers under different administrative domains. Leveraging this type of platform is challenging because resources are heterogeneous, dynamic, and under different administrative policies. Fortunately, several Grid infrastructure projects [13-17] are available to facilitate running an application across different administrative domains. Our implementation of off-line parallel tomography, GTOMO [4], uses services from the Globus toolkit [13] for remote job management, security, and interprocess communication. To address the heterogeneous, dynamic properties of a Grid [5], we use an application-level scheduling strategy.

An AppLeS (application-level scheduler) [8] integrates with the target application to develop a schedule for deploying the application in a Grid environment. The scheduler makes predictions of the performance the application may experience on prospective resources at execution time. Using these predictions, a potentially performance-efficient schedule for the application is identified and deployed [18-20,4]. In the case of GTOMO, an embarrassingly parallel application, it is natural to use self-scheduling [21]. We opted for a greedy work queue algorithm where computation is assigned to processors as soon as they become available. The algorithm is also coupled with a resource selection strategy that co-allocates the execution of parallel tomography over workstations and immediately available supercomputer nodes.

The architecture of GTOMO is displayed in Fig. 2. A multi-threaded reader process reads input data (called sinograms) from disk and sends that data to ptomo processes. Each ptomo process performs a part of the reconstruction and sends reconstructed slices to a writer process which writes the data back to disk. The figure also displays a driver process which controls the work queue and coordinates the interactions among all the different processes.

\subsection{On-line Parallel Tomography}

Currently, NCMIR users run tomography on their data after they collect it from the electron microscope (off-line). Upon visualization of their data, they sometimes discover a misconfiguration of the microscope or might find a more interesting area of the specimen to study. In such cases, users must modify the microscope parameters and acquire a new dataset. This requires at least 45 additional minutes and increases beam damage to the specimen [22]. It would therefore be extremely useful to provide feedback to the user by computing incremental tomograms during the acquisition process.

\subsubsection{Extensions to GTOMO}

To incrementally compute a tomogram, we update the $i^{\text {th }}$ slice of the tomogram with the $i^{\text {th }}$ scanline of each projection as it is acquired from the electron mi- 


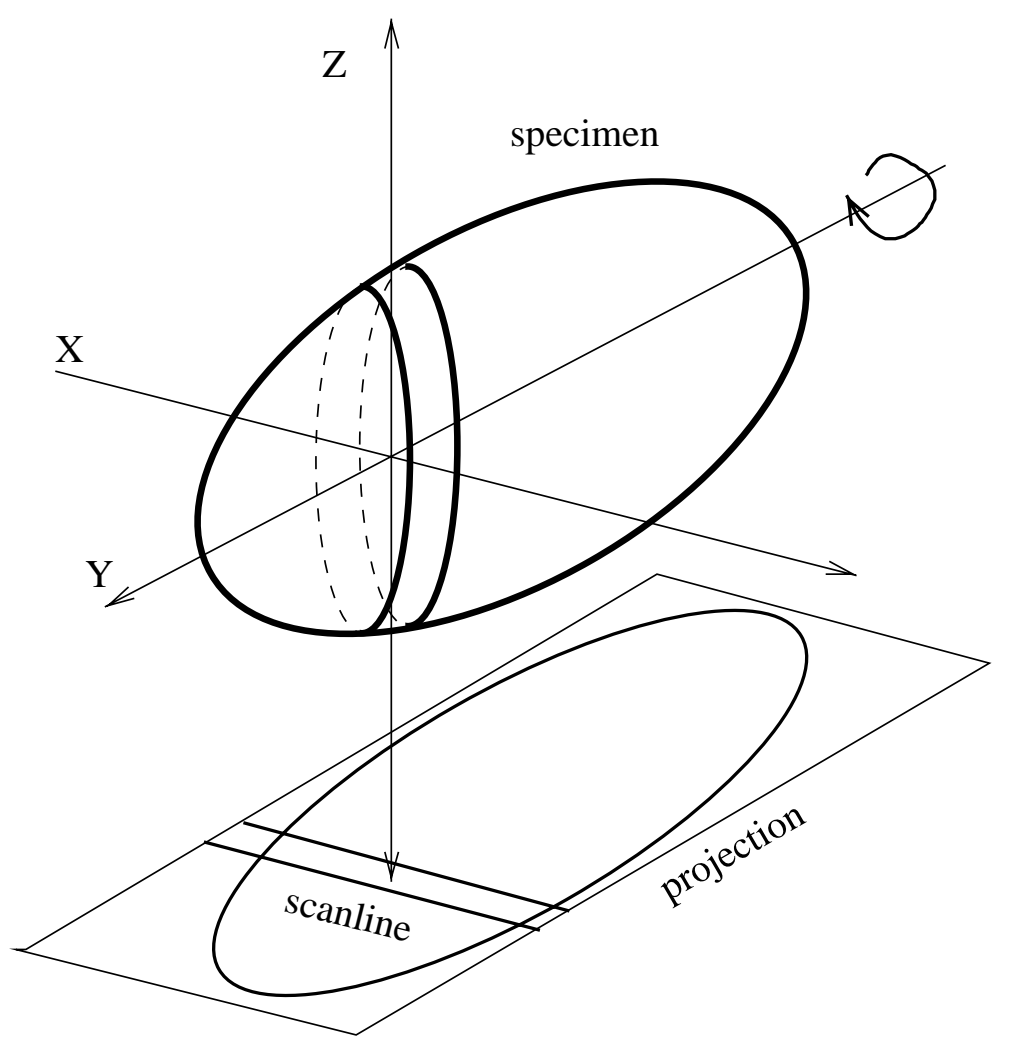

Fig. 1. Parallelism of tomography (adapted from [3]). The information required to reconstruct the $i$ th $\mathrm{X}-\mathrm{Z}$ slice is the $i$ th scanline from all projections.

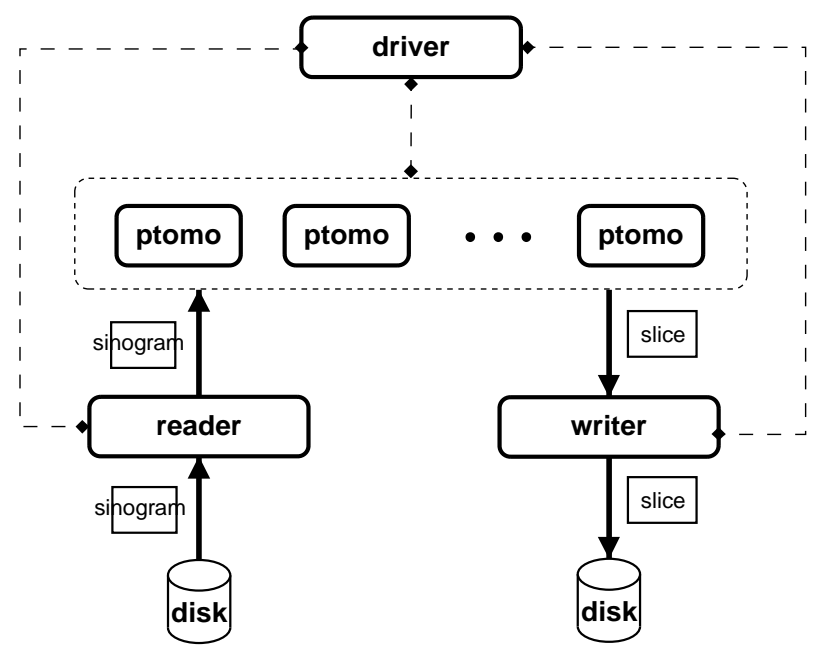

Fig. 2. Off-line GTOMO architecture.

croscope. Achieving this in real-time requires a reconstruction technique that is fast and augmentable. An augmentable technique allows each successive computation to build upon the previous computation without repeating work. Fortunately, the R-weighted backpro- jection technique [10] fulfills both requirements.

The implementation of the R-weighted backprojection method as an augmentable technique mandates a modification of the GTOMO application. It requires that we send the $i^{t h}$ scanline from each projection to 
the same ptomo process so that it may process the information into the same slice. Therefore, the work queue approach described in Section 2.2 is no longer viable and we replace it with a static work allocation strategy. We leave rescheduling for future work.

The structure of on-line GTOMO is shown in Fig. 3. The electron microscope sends a projection to the preprocessor every $a$ seconds. The preprocessor divides the projection into sections, where each section contains multiple scanlines. The scanlines in each section will be processed in parallel by ptomo processes. All ptomos will periodically send their slices to the writer in order to update the tomogram. A visualization program will then display updated tomograms to the user. The driver coordinates interactions among all other processes.

\subsubsection{Tunability}

Because on-line parallel tomography is resourceintensive, we want to consider its execution on dynamic Grids with differing resource capabilities. Therefore, we have designed our implementation of on-line parallel tomography to be tunable. A tunable application is characterized by the availability of alternate configurations, where each configuration corresponds to a different execution path and resource usage [7]. In this work, tunability allows us to express trade-offs between tomogram resolution and the frequency of tomogram updates. We next illustrate its usage through an example.

We define the acquisition period, $a$, as the time to acquire a projection from NCMIR's electron microscope. NCMIR is currently targeting an acquisition period of 45 seconds; therefore, we use this value throughout our work. Consider a $(61,2048,2048,600)$ experiment (see Section 2.1) which yields a tomogram of about 9.4 GB. If we place our writer on a machine with an observable bandwidth of $100 \mathrm{Mb} / \mathrm{s}$, it will take $768 \mathrm{sec}-$ onds to transfer the whole tomogram. To avoid overloading the network, we send only one tomogram at a time. So for this experiment, successive tomograms should be sent at least 768 seconds apart. Since a projection is processed every 45 seconds, we can send a tomogram every $\left\lceil\frac{768}{45}\right\rceil=18$ projections. We call each send a refresh and say that the number of processed projections per refresh is 18 . The period of the refresh is $18 \times 45=810$ seconds, approximately 14 minutes. After surveying the requirements of NCMIR users, it appears that no user would tolerate a refresh periods which is over 10 minutes.

Now, suppose we reduce the resolution of the projections by a factor of 2 in each dimension. For the time being we consider a simple averaging strategy [23]. The tomogram would then be $1.2 \mathrm{~GB}, 8$ times smaller. Thus, it would take only 96 seconds to transfer each tomogram, yielding an acceptable refresh period of 135 seconds. Note that further reductions are possible but in order to yield a sufficiently detailed tomogram for NCMIR users, the projections should not be reduced beyond $256 \times 256$ pixels.

Two parameters, therefore, determine the quality of on-line parallel tomography: reduction factor and projections per refresh. The reduction factor $(f)$ is a scalar value that specifies a reduction of the size of a projection in each dimension. If we have a projection of size $x \times y$, after reduction, we will have a projection of size $\frac{x}{f} \times \frac{y}{f}$. Increasing the reduction factor decreases the number of slices and the amount of computation and communication per slice. The projections per refresh $(r)$ parameter refers to the number of new projections processed into each successive tomogram refresh. An increase in $r$ reduces the frequency of refreshes sent to the user and thus reduces the amount of communication.

\section{Scheduling}

We have defined the pair $(f, r)$ that determines the configuration of the application. If enough resources were available, users would always choose $(1,1)$ which would result in the highest resolution tomogram being refreshed at the highest frequency possible. Given insufficient resources, in practice users need to choose an alternate configuration when the ideal configuration is infeasible. This choice depends on each user's requirements; therefore our scheduler aims at assisting users in selecting an appropriate configuration. At runtime, the scheduler discovers which pairs are feasible and allows users to chose a pair that best fits their requirements for execution. The process of discovering feasible pairs is described in the following subsections.

\subsection{Constraints for on-line tomography}

We evaluate on-line parallel tomography as a soft real-time application, one that is characterized by the execution of tasks which have soft deadlines [24]. That is, the usefulness of the task decreases as the tardiness of the task increases [24,25]. Given the discussion in Section 2.3, our soft deadlines are:

(i) the computation time of one projection is less than the acquisition period, 


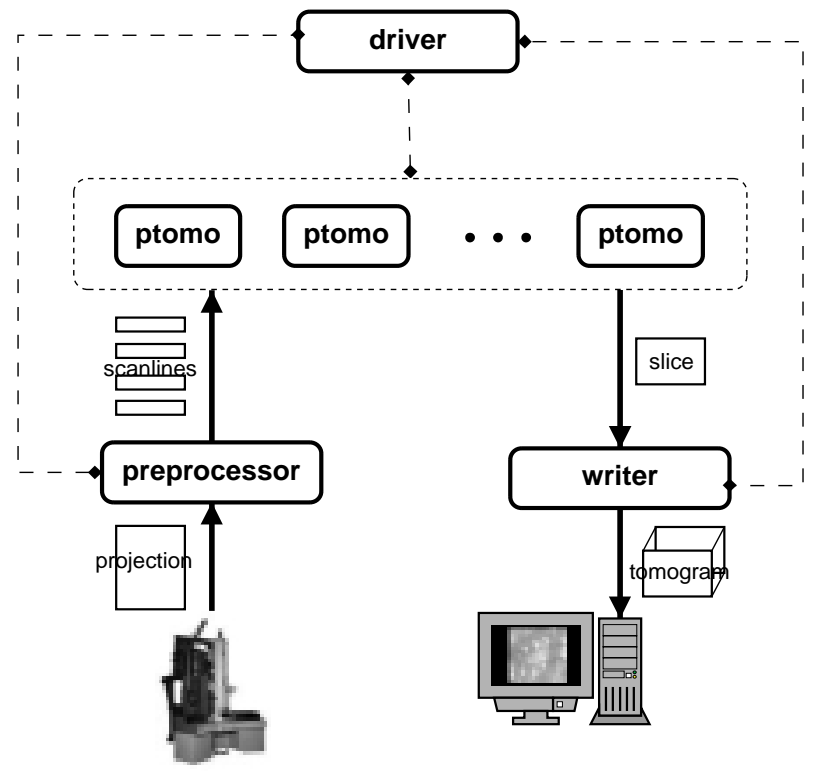

Fig. 3. On-line GTOMO architecture.

(ii) the transfer time of a tomogram is less than the refresh period.

Our goal is to find a configuration of the application for which all deadlines are met.

Consider a tomography experiment $(p, x, y, z)$ and a pair $(f, r)$. Our scheduler must allocate work to resources. For a set of compute resources $M$, we define a work allocation as a set $W$ :

$$
W=\left\{w_{m}: m \in M\right\}
$$

where $w_{m}$ is the number of tomogram slices allocated to processor $m$.

We have the two following constraints:

$$
\begin{aligned}
& \forall m \in M w_{m} \geqslant 0 \\
& \sum_{m \in M} w_{m}=\frac{y}{f}
\end{aligned}
$$

since there are a total of $\frac{y}{f}$ tomogram slices to compute. In the following two sections we derive constraints for the computation deadline and the communication deadline.

\subsection{Computation deadline}

The soft deadline for computation can be written as:

$$
\forall m \in M \quad T_{\text {comp }}(m) \leqslant a,
$$

where $T_{\text {comp }}(m)$ is the time to backproject a single projection into $w_{m}$ slices on processor $m$ and $a$ is the acquisition period.
A simple analysis of the augmentable R-weighted backprojection algorithm used for the tomographic reconstruction shows that

$$
T_{\text {comp }}(m) \approx t p p_{m} \times \frac{x}{f} \times \frac{z}{f} \times w_{m},
$$

where $t p p_{m}$ is the time to process a single pixel of a tomogram slice on dedicated processor $m$.

We model two types of compute resources: timeshared workstations and space-shared supercomputers. Let $T S R$ be the set of time-shared workstations and $S S R$ be the set of space-shared supercomputers such that

$$
M=T S R \cup S S R .
$$

On a time-shared workstation,

$$
T_{\text {comp }}(m)=\frac{t p p_{m}}{c p u_{m}} \times \frac{x}{f} \times \frac{z}{f} \times w_{m}
$$

where $c p u_{m}$ is the fraction of CPU available on processor $m$. In practice we obtain a prediction for the value of $c p u_{m}$ from the Network Weather Service (NWS) [26]. Likewise, for a space-shared supercomputer,

$$
T_{\text {comp }}(m)=\frac{t p p_{m}}{u_{m}} \times \frac{x}{f} \times \frac{z}{f} \times w_{m}
$$

where $u_{m}$ is the number of unused nodes on $m$ that are immediately available for execution. As done in [4], we opt to use MPP processors only when they are immediately available. This avoids unpredictable queue waiting times which are prohibitive for our scenario of a soft real-time application. In practice we can obtain $u_{m}$ from batch schedulers such as the Maui Scheduler [27]. 


\subsection{Communication deadline}

The soft deadline for data transfers can be written as:

$$
\forall m \in M \quad T_{\text {comm }}(m) \leqslant r \times a,
$$

where $T_{\text {comm }}(m)$ is the time for $m$ to transfer $w_{m}$ slices to the writer.

Given that tomogram slices are generally several megabytes in size, we use the following approximation of the equation in [28]:

$$
T_{\text {comm }}(m) \approx \frac{w_{m} \times\left(\frac{x}{f} \times \frac{z}{f} \times s z\right)}{B_{m}},
$$

where $s z$ is the number of bits used to represent a pixel and $B_{m}$ is the bandwidth between processor $m$ and the writer. We can obtain predictions for $B_{m}$ from the NWS [26].

Note this model assumes a fully connected network where each processor has a dedicated link to the writer. However resources are usually connected by way of shared network links $[29,30]$. Therefore, we incorporate network topology information into our model in order to determine a more effective work allocation. We group resources into subnets, where a subnet contains a set of compute resources which share a network link to the writer. Let $S$ be the set of subnets such that

$$
\bigcup_{S_{i} \in S} S_{i}=M
$$

and $S_{i}$ is a subnet. In practice, the subnet groupings in $S$ can be obtained using a tool like ENV [31].

The following additional transfer constraint can then be introduced into our model:

$$
\forall S_{i} \in S \quad T_{\text {comm }}\left(S_{i}\right) \leqslant r \times a
$$

where $T_{\text {comm }}\left(S_{i}\right)$ is the time for all compute resources in $S_{i}$ to transfer $\sum_{m \in S_{i}} w_{m}$ slices to the writer. For Equation 10, we can now write:

$$
T_{\text {comm }}\left(S_{i}\right) \approx \frac{\left(\sum_{m \in S_{i}} w_{m}\right) \times \frac{x}{f} \times \frac{z}{f} \times s z}{B_{S_{i}}}
$$

where $B_{S_{i}}$ is the capacity of the subnet link. Note that because we assume a heterogeneous network, Equation 13 complements Equation 10 rather than invalidating it.

We do not introduce any constraints into our model for input data transfers (i.e., projection data sent from the preprocessor to the ptomos). The input data is one order of magnitude smaller than the output data and its transfer time is amortized into the acquisition period.

Finally, our scheduling algorithm assumes that the user provides bounds on the tunable parameters, hence the last couple of constraints:

$$
\begin{aligned}
& f_{\text {min }} \leqslant f \leqslant f_{\text {max }} \\
& r_{\text {min }} \leqslant r \leqslant r_{\text {max }}
\end{aligned}
$$

All our constraints are summarized in Fig. 4.

\subsection{Scheduling and tuning: an optimization problem}

Our goal is to present the user with a set of feasible pairs $(f, r)$ (i.e., pairs for which there exists a work allocation that satisfies the constraints in Fig. 4). One approach is exhaustive search: for each pair $(f, r)$, one can solve the system in Fig. 4 to find a possible work allocation. A more efficient approach is to solve two optimization problems:

(i) fix $f$ and minimize $r$,

(ii) fix $r$ and minimize $f$,

where both problems are subject to the constraints in Fig. 4. This approach can be easily extended to a larger number of tuning parameters whereas exhaustive search does not scale (see Section 6). Finally, an added advantage of this approach is that it filters out suboptimal triples. For example, suppose that triples $(1,1)$ and $(1,2)$ are feasible. We assume that users would alway choose $(1,1)$ over $(1,2)$.

We describe here how these optimization problems can be solved. First, we see that optimization problem (i) becomes linear upon substitution of $f$. This is a clear advantage as there are numerous linear programming solvers available for download [32]. However, for (ii) the system remains nonlinear. While nonlinear programming solvers are available [33], as a first approach, we opt to use a more simple technique. We exploit the discreteness and small range of $f$ to reduce the nonlinear program to multiple linear programs using substitution. All linear programs are then solved and the optimal solution is chosen. For our linear solver, we have chosen to use the lp_solve package [34].

Ideally, our optimal solution would be found by formulating the linear program as an integer program. An integer program is a linear program where all variables are constrained to be integers [35]. However, integer programs are harder to solve than linear programs [32]. Our experiments indicate that a mixedinteger approach, where $w_{m}$ is expressed as continuous variables and all others as integer variables is efficient. 


$$
\begin{aligned}
& \forall m \in M \quad w_{m} \geq 0 \\
& \sum_{m \in M} w_{m}=\frac{y}{f} \\
& \forall m \in T S R \quad \frac{t p p_{m}}{c p u_{m}} \times \frac{x}{f} \times \frac{z}{f} \times w_{m} \leq a \\
& \forall m \in S S R \quad \frac{t p p_{m}}{u_{m}} \times \frac{x}{f} \times \frac{z}{f} \times w_{m} \leq a \\
& \forall m \in M \quad \frac{w_{m} \times\left(\frac{5}{7} \times \frac{\pi}{j} \times 4\right)}{B_{m}} \leq r \times a \\
& \forall S_{i} \in S \frac{\left(\sum_{m \in S_{i}} w_{m}\right) \times \frac{x}{f} \times \frac{z}{f} \times 4}{B_{S_{i}}} \leq r \times a \\
& f_{\min } \leq f \leq f_{\max } \\
& r_{\min } \leq r \leq r_{\max }
\end{aligned}
$$

Fig. 4. Constraints for on-line parallel tomography.

The drawback of this approach is that the values found for $w_{m} \in \mathbf{R}$ must be rounded to integers (since it does not make sense to allocate fractional slices to ptomos). Therefore, the result is an approximate solution. We evaluate its effectiveness in Section 4.3.1.

\section{Experimental results}

In this section, we evaluate the on-line GTOMO scheduler using simulation. Section 4.1 describes our simulator and Section 4.2 describes our simulated Grid environment. We then show two sets of results. In Section 4.3, we show that using dynamic system information improves scheduler performance. In the second set of results, described in Section 4.4, we demonstrate that tunability is a fundamental concept for production on-line parallel tomography runs in a Grid environment.

\subsection{Simulator Description}

In order to evaluate our scheduler performance, we execute the application with multiple scheduling strategies under the same environmental conditions. However, achieving repeatable environmental conditions is not possible in a Grid environment [5]. One approach is to run back-to-back experiments $[4,19,20]$. However, this is not appropriate for tomography given the long makespan of the application. Therefore, we employ simulation [36]. We wrote a simulator using Simgrid [37], a discrete-event simulation toolkit which provides APIs for studying scheduling algorithms in distributed systems. Simgrid allows us to implement a discrete-event simulator and provides a notion of tasks (e.g., computations, data transfers) and resources (e.g., processors, network links). Tasks can have dependencies among them and are scheduled on resources. Resources behaviors are modeled by service rates that can be modeled using traces from real resources (e.g., CPU availability, network link bandwidth ). Such traces are commonly available by existing resource monitoring tools such as the NWS [26]. Furthermore, Simgrid makes it possible to create arbitrary resource interconnect topologies. The Simgrid approach has been verified in [37] and has been used to evaluate scheduling algorithms for parameter sweep applications [36,38]. Similar trace-based resource simulation approaches have also been applied in projects such as Bricks [39].

In our simulator, we model four types of tasks based on profile information from the application:

1. acquire: acquire a projection from the microscope

2. scanline transfer: send a scanline from the preprocessor to a ptomo

3. backproject computation: backproject a scanline to a slice

4. slice transfer: send a slice from a ptomo to the writer

For the simulation of a single run, there are $p$ acquires. For each acquire, there are $\frac{y}{f}$ scanline transfers and $\frac{y}{f}$ backprojection computations. Given the value 


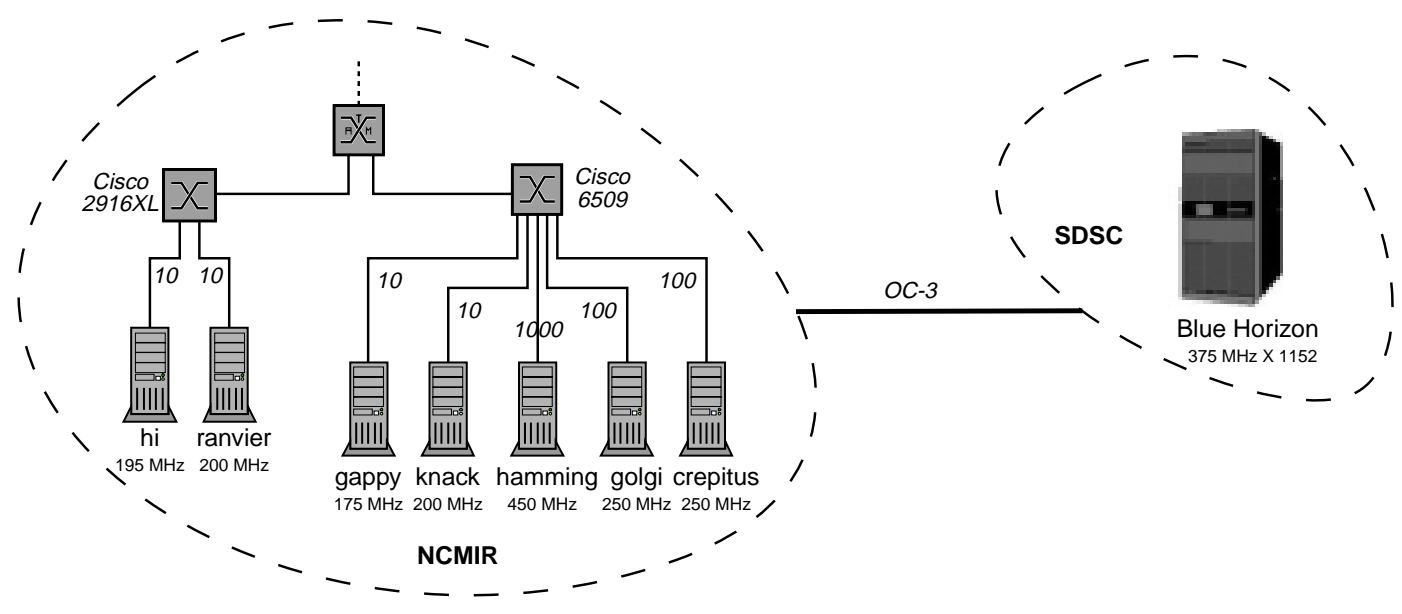

Fig. 5. NCMIR Grid topology.

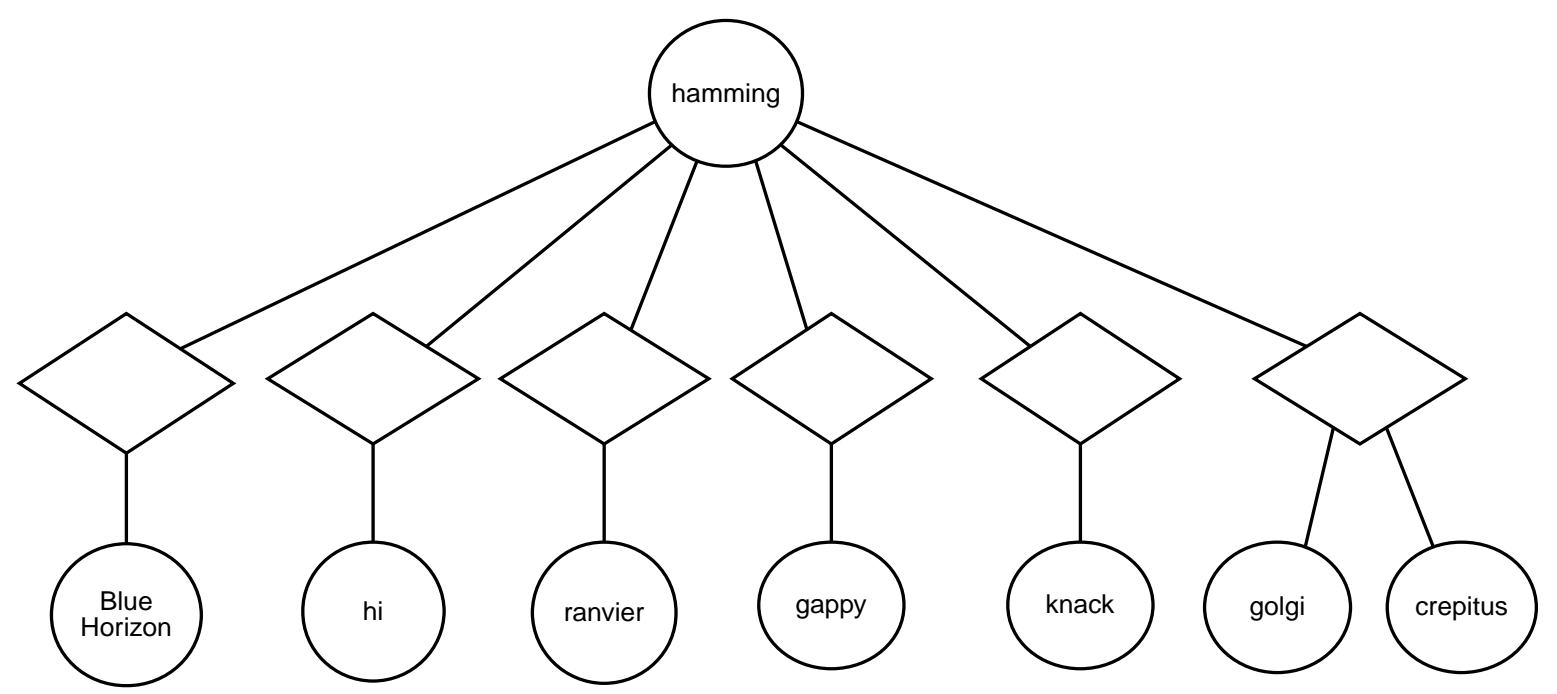

Fig. 6. ENV representation of NCMIR Grid topology.

of the refresh period, $r$, there can also be $\frac{y}{f}$ slice transfers following the backprojection computations. Resources are modeled as a Grid containing multi-user workstations and space-shared supercomputers.

\subsection{Case Study: NCMIR Grid}

We simulate a set of resources modeled after a subset of the real computational environment at NCMIR. The real network topology is shown in Fig. 5. It is composed of a cluster of 7 workstations at NCMIR and the Blue Horizon SP/2 at the San Diego Supercomputer Center (SDSC). Since our goal is to develop a method that is applicable in any environment, we use a tool to automatically discover the topology and build a rele- vant model that can be used for scheduling. In this work we used ENV [31]. Figure 6 shows the ENV representation of the topology relative to hamming. The machine hamming was used both as the preprocessor and writer machine because it had the highest bandwidth capacity. Note that due to the switched network and hamming's $1 \mathrm{~Gb} / \mathrm{s}$ NIC, almost all machines appeared as if they had dedicated network links to hamming. The exceptions were golgi and crepitus which both have $100 \mathrm{Mb} / \mathrm{s}$ NICs. In this case, the ENV tool detected some network interference at the switch. We therefore modeled golgi and crepitus as sharing the same network link in our simulations. Note that at the time of the experiments we did not have any knowledge of the network topology within SDSC. This further jus- 


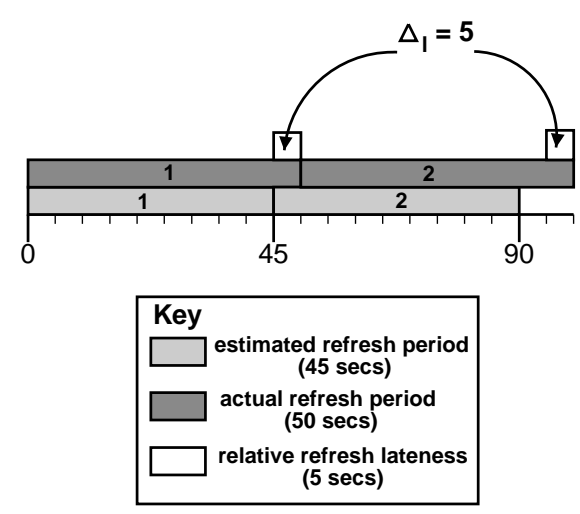

Fig. 7. Example timeline (partial) of an on-line parallel tomography experiment. The estimated refresh period (45 seconds) is shown in light gray while the actual refresh period ( 50 seconds) is shown in dark gray. The $\Delta_{\text {f }}$ for both the first and second refresh is 5 seconds.

Table 1

Summary statistics for the CPU availability traces

\begin{tabular}{lccccc}
\hline & mean & std & cv & $\min$ & $\max$ \\
\hline gappy & 0.996 & 0.016 & 0.016 & 0.815 & 1.000 \\
golgi & 0.700 & 0.231 & 0.330 & 0.109 & 0.939 \\
knack & 0.896 & 0.118 & 0.132 & 0.377 & 0.986 \\
crepitus & 0.925 & 0.060 & 0.065 & 0.401 & 0.940 \\
ranvier & 0.981 & 0.042 & 0.043 & 0.394 & 0.994 \\
hi & 0.832 & 0.207 & 0.249 & 0.426 & 1.000 \\
\hline
\end{tabular}

tifies the use of a tool like ENV as network topology information is not always available and changes over time. ENV gives us a way to model possible contention among resources that share network links to the writer process.

To model load on NCMIR workstations, we collected CPU availability traces using the NWS from May 19th until May 26th 2001. During this time, we also collected a node availability trace from Blue Horizon to model its load using the Maui Scheduler command showbf [27]. Similarly, bandwidth traces were collected from all machines to hamming. The sample period for both CPU availability and bandwidth were set to the NWS defaults, 10 and 120 seconds respectively. The sample period for the Blue Horizon traces was 5 minutes. Summary statistics for the traces are displayed in Tables 1, 2, and 3. For each trace, the table shows the mean (mean), the standard deviation ( $s t d)$, the coefficient of variance $(\mathrm{cv})$, the minimum ( $\mathrm{min})$, and the maximum (max) trace values.

All the results hereafter were obtained with our Simgrid-based simulator using an acquisition period of 45 seconds (see Section 2.3.2).

\subsection{Work allocation results}

We evaluate the performance of the tomography application in terms of soft deadline violations (see Sec-
Table 2

Summary statistics for the bandwidth traces $(\mathrm{Mb} / \mathrm{s})$

\begin{tabular}{lrrrrr}
\hline & \multicolumn{1}{c}{ mean } & \multicolumn{1}{c}{ std } & cv & min & \multicolumn{1}{c}{$\max$} \\
\hline gappy & 8.335 & 0.778 & 0.093 & 3.484 & 9.145 \\
knack & 5.966 & 2.355 & 0.395 & 0.616 & 9.005 \\
golgi/crepitus & 70.223 & 19.657 & 0.280 & 3.104 & 81.361 \\
ranvier & 3.613 & 0.242 & 0.067 & 0.620 & 9.005 \\
hi & 7.820 & 2.230 & 0.285 & 0.353 & 13.074 \\
horizon & 32.754 & 7.009 & 0.214 & 0.180 & 41.933 \\
\hline
\end{tabular}

Table 3

Summary statistics for node availability trace

\begin{tabular}{cccccc}
\hline & mean & $\mathrm{std}$ & $\mathrm{cv}$ & $\min$ & $\max$ \\
\hline Blue Horizon & 31.1 & 48.3 & 1.5 & 0.0 & 492.0 \\
\hline
\end{tabular}

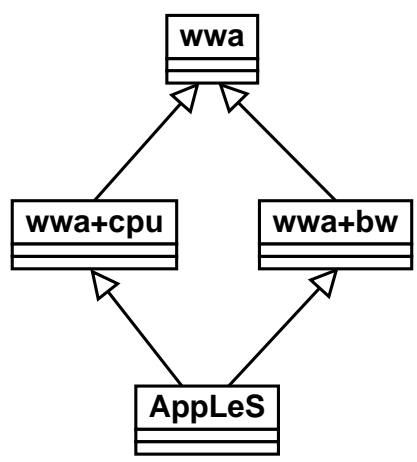

Fig. 8. UML diagram describing scheduler characteristics.

tion 3.1). Our performance metric is relative refresh lateness $\left(\Delta_{l}\right)$, that is the difference between the predicted and actual refresh times with respect to the lateness of the previous refresh. Therefore, low $\Delta_{l}$ infer better real-time execution. We illustrate $\Delta_{l}$ with an example shown in Fig. 7.

We compare our scheduler, AppLeS, to three schedulers: wwa, wwa+cpu, and wwa+bw. Weighted work allocation, or wwa, corresponds to a simple strategy 


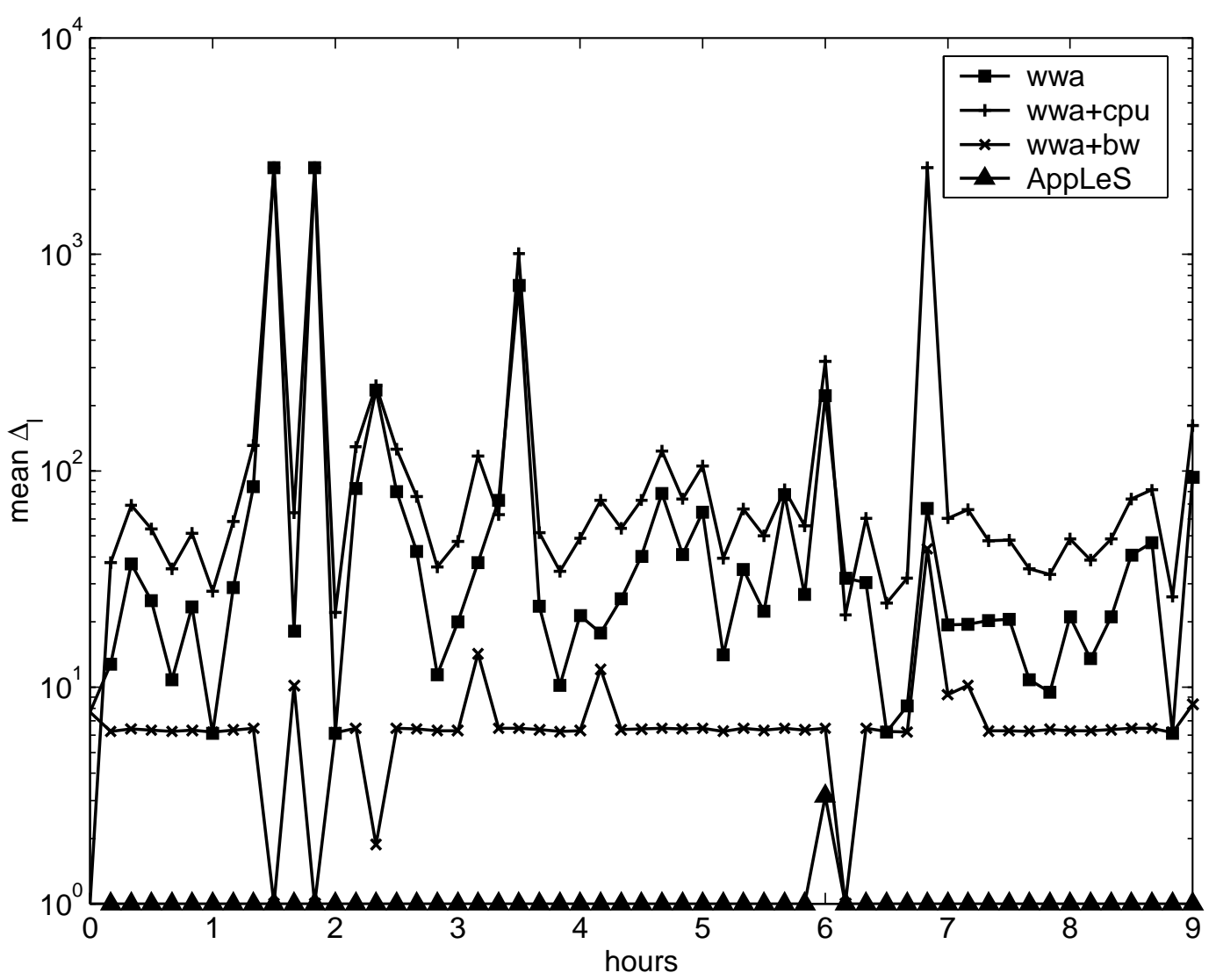

Fig. 9. The mean $\Delta_{l}$ for the simulation period of May 22, 2001 from 8:00 A.M.-5:00 P.M. is plotted for each scheduler.

that a user might employ: it performs work allocation based only on the relative processor benchmark of the application in dedicated mode. The second scheduler, wwa+cpu, assumes that compute resources are shared among multiple users. It extends wwa by utilizing dynamic CPU load information. This corresponds to users that might run a system command such as uptime to find out CPU availability before executing their application. The wwa+bw scheduler assumes only dynamic bandwidth information and no CPU load information. The AppLeS scheduler, as described in Section 3, assumes that both compute and network resources are shared among multiple users. The relationship between the four schedulers is illustrated using an UML diagram in Fig. 8.

We conducted two distinct sets of experiments: partially trace-driven simulations and completely tracedriven simulations. In both sets of simulations, we fixed the pair $(f, r)$ and use the schedulers to determine work allocation. The following results were obtained using a $1 k \times 1 k$ dataset. For each set we simulate 1004 runs of the application throughout the week starting every
10 minutes. Simulations were also run for a $2 k \times 2 k$ dataset but since the dataset was always reduced by a factor of 2 , the simulation results were identical to the $1 k \times 1 k$ set.

\subsubsection{Partially trace-driven simulations}

In this set of experiments, we simulated runs where the schedulers had access to perfect load predictions. This represents the optimal running environment for the schedulers since the performance predictions made at the beginning of execution are valid throughout the entire execution. At the start of each simulation, we used the trace to determine a constant resource load for the duration of the simulation. This allows us to test our scheduling strategy in different Grid conditions, but without dynamic Grid resource behaviors.

Figure 9 shows the simulation results of the $1 k \times 1 k$ experiment using the traces collected on May 22, 2001 from 8:00 A.M. to 5:00 P.M. We plot the mean relative refresh lateness for each scheduler over the nine hour simulation period. In these simulations, it is clear that the AppLeS scheduler outperforms all the other sched- 


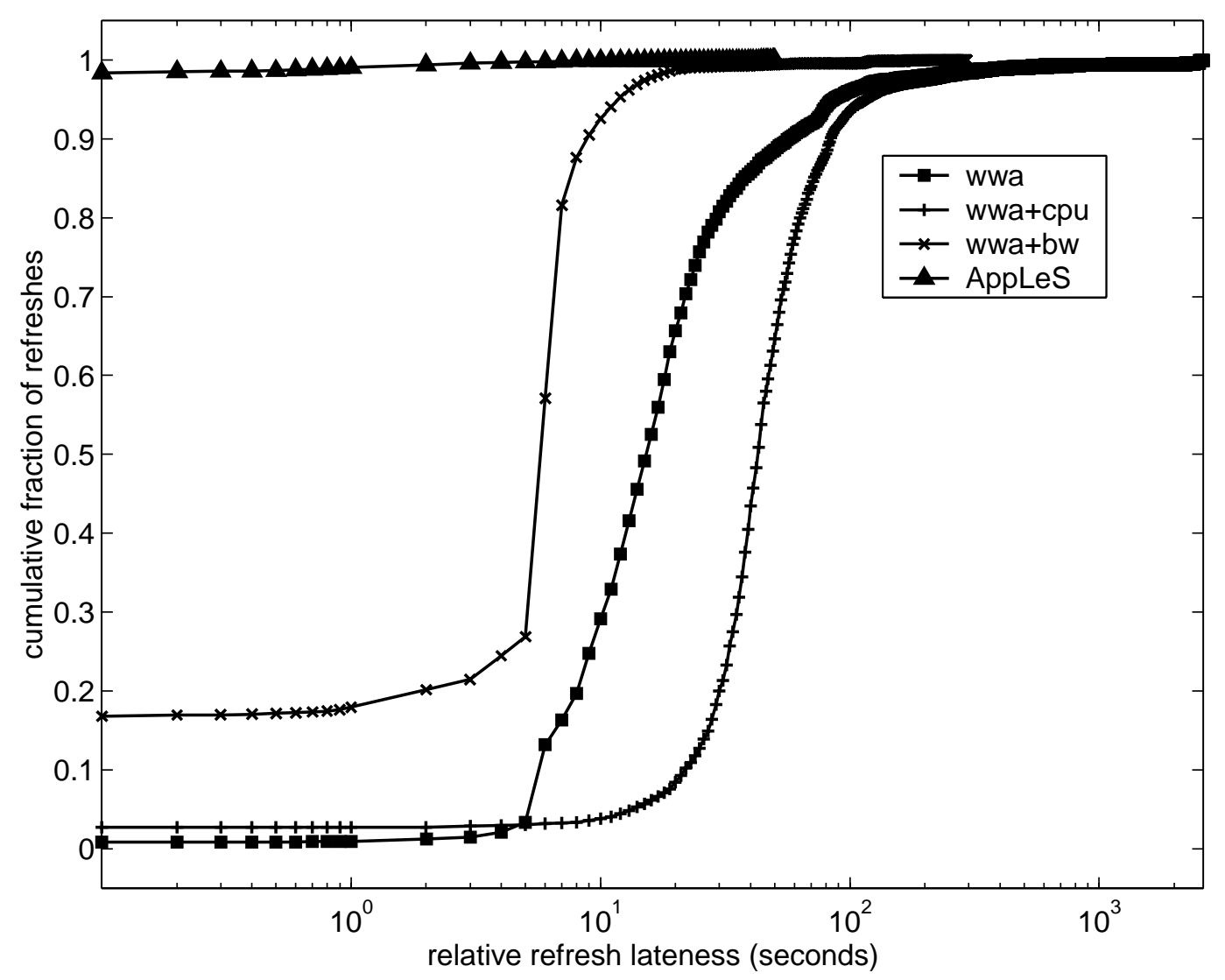

Fig. 10. Partially trace-driven simulations: the cumulative distribution functions of $\Delta$ for each scheduler.

ulers. It is followed by the wwa+bw scheduler which outperforms both the wwa and wwa+cpu schedulers indicating that communication is the dominant factor in application performance. Surprisingly, we see that the wwa scheduler appears to do better than wwa+cpu. Upon further investigation, we see that the wwa scheduler allocates most of its work to crepitus, one of the machines with high bandwidth capacity to hamming (see Table 2). Conversely, the wwa+cpu scheduler allocated a higher amount of work to Blue Horizon because it detected a drop in CPU availability on crepitus. While Blue Horizon had higher CPU availability, it had a lower bandwidth capacity to hamming. Therefore, wwa did better than wwa+cpu. Thus in these simulations, CPU availability information was not useful unless it was accompanied with bandwidth information (i.e., AppLeS outperforms wwa+bw). We are currently running simulations on different types of Grids where wwa+cpu outperforms wwa.

Figure 10 shows the results of simulating the $1 k \times 1 k$ dataset throughout the whole week of traces. For each scheduler, we plot the cumulative distribution function
Table 4

Average deviation from best scheduler based on cumulative $\Delta_{l}$

\begin{tabular}{lrrrrr}
\hline scheduler & \multicolumn{2}{c}{$\begin{array}{c}\text { partially } \\
\text { trace-driven }\end{array}$} & & \multicolumn{2}{c}{$\begin{array}{c}\text { completely } \\
\text { trace-driven }\end{array}$} \\
\cline { 2 - 3 } \cline { 5 - 6 } & \multicolumn{1}{c}{ avg } & std & & avg & std \\
\hline wwa & 783.70 & 715.63 & & 237.01 & 190.22 \\
wwa+cpu & 1116.17 & 604.16 & & 544.59 & 305.12 \\
wwa+bw & 159.04 & 159.56 & & 74.21 & 93.11 \\
AppLeS & 0.08 & 2.49 & & 49.94 & 96.33 \\
\hline
\end{tabular}

of $\Delta_{l}$. A point $(x, y)$ on the graph represents that $y$ percent of the refreshes were less than or equal to $x$ seconds late. Here, we see that $2 \%$ of the refreshes arrived late for the AppLeS scheduler due to the approximation strategy described in Section 3.4. $1 \%$ of these refreshes were less than or equal to 1 second late, $0.9 \%$ were less than or equal to 10 seconds late, and the remaining $0.1 \%$ refreshes were less than or equal to 50 seconds late. In these cases, low bandwidth affected the impact of rounding (to get an approximate solution). In particular, the case where $\Delta_{l}$ was approximately 40 seconds late, the bandwidth to the machine hi was quite low at approximately $444 \mathrm{~Kb} / \mathrm{s}$. 


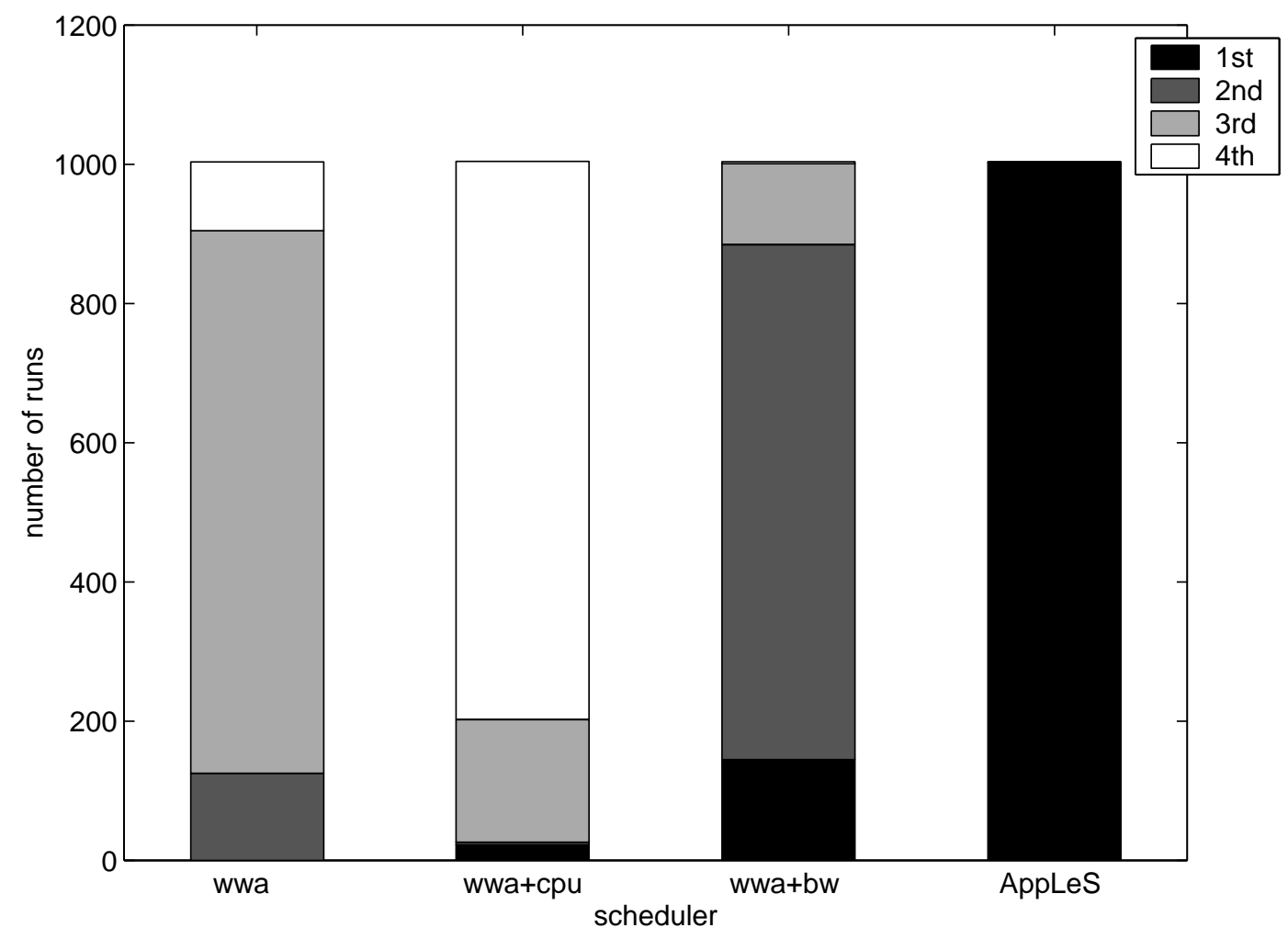

Fig. 11. Partially trace-driven simulations: scheduler ranking based on cumulative $\Delta$.

To compare the simulation results for the schedulers on a run-to-run basis, we plotted the number of times each scheduler ranked first, second, third, and fourth place based on cumulative $\Delta_{l}$ (i.e., $\sum \Delta_{l}$ for each run) in Fig. 11. Ranking for this graph was decided as follows:

(i) For a single run, scheduler $i$ received a rank $k$ if $k-1$ schedulers beat it.

(ii) For a single run, if more than one scheduler had the the same cumulative relative refresh lateness, they received the same rank.

To measure the magnitude of difference from the best, we calculated the average deviation (avg) from best scheduler based on cumulative $\Delta_{l}$ for each run. We also calculate the standard deviation (std). The results are displayed in the first and second columns of Table 4.

\subsubsection{Completely trace-driven simulations}

In this set of experiments, we used traces to determine resource load variation throughout simulation. Therefore, these simulations are completely tracedriven. Consequently, the initial load predictions may be imperfect throughout the simulated period. In other words, these simulation results show the impact of dynamic Grid resource behavior on scheduling.

Figure 12 shows the results of the simulations in a cumulative distribution function plot. Comparing this to the previous set of simulations, we see how imperfect predictions degrade the performance of the AppLeS scheduler. Here $42.9 \%$ of the refreshes arrive late compared to $2 \%$ in the partially trace-driven simulations. Although, we note that only $3.4 \%$ of the refreshes arrive later than 600 seconds (the upper bound of tolerance for NCMIR users). We also plotted the scheduler rankings in Fig. 13. These results show that the AppLeS scheduler was in first place $55 \%$ of the time compared to almost $100 \%$ in the partially tracedriven simulations. The average deviations from best scheduler are displayed in the third column of Table 4 and the standard deviations are displayed in the fourth column.

Comparing the performance of the AppLeS to the other schedulers, we see that it ranked first in more runs than the other schedulers. Furthermore, on average the AppLeS scheduler showed a 24.27 second improvement in cumulative $\Delta_{l}$ per run over the wwa+bw scheduler (see Table 4). We are currently running fur- 


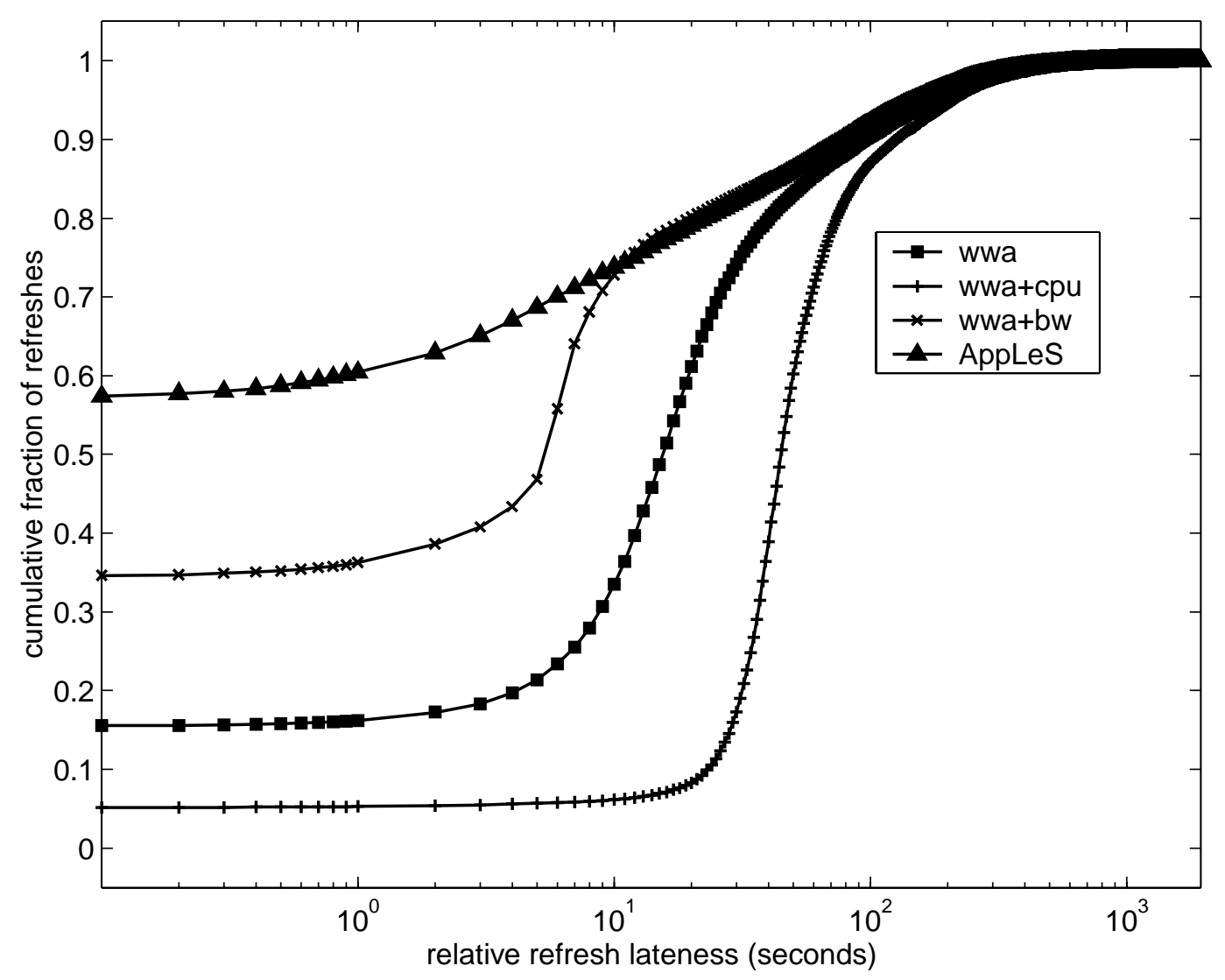

Fig. 12. Completely trace-driven simulations: the cumulative distribution functions of $\Delta$ for each scheduler.

ther simulations on Grids with differing levels of dynamic resource availability to evaluate the effects of dynamic Grid behavior on scheduler performance. As mentioned in Section 2.3.1, the benefit of rescheduling (to cope with imperfect predictions) is left for future work.

\subsection{Evaluation of tunability}

In Section 2.3.2, we motivated the design of on-line parallel tomography as a tunable application for dynamic Grid environments. In this section, we assess the usefulness of tunability. We say that tunability is useful if changing the configuration at run-time (from the previous configuration) results in a better configuration for the user and/or better real-time execution than not changing the configuration. We study how the configuration of on-line parallel tomography would change for a user running back-to-back experiments during a one-week period at NCMIR (see Section 4.2).

We consider two different on-line parallel tomography experiments:

$$
\begin{aligned}
& E_{1}=(45,61,1024,1024,300), \\
& E_{2}=(45,61,2048,2048,600) .
\end{aligned}
$$

As described in Section 2.2, these two experiments are representative of the size of experiments run by NCMIR users and correspond to datasets collected from $1 k \times 1 k \mathrm{CCD}$ and $2 k \times 2 k \mathrm{CCD}$ cameras respectively. Based on NCMIR user preferences (as discussed in Section 2.3.2), we set the following constraints for $E_{1}$ experiments:

$$
\begin{aligned}
& 1 \leqslant f \leqslant 4 \\
& 1 \leqslant r \leqslant 13,
\end{aligned}
$$

and for $E_{2}$ experiments:

$$
\begin{aligned}
& 1 \leqslant f \leqslant 8 \\
& 1 \leqslant r \leqslant 13 .
\end{aligned}
$$

We simulated scheduler decisions every 10 minutes throughout the simulated week leading to 1004 reconstructions for each experiment type. The range of $(f, r)$ pairs found by the AppLeS scheduler for the $E_{1}$ exper- 


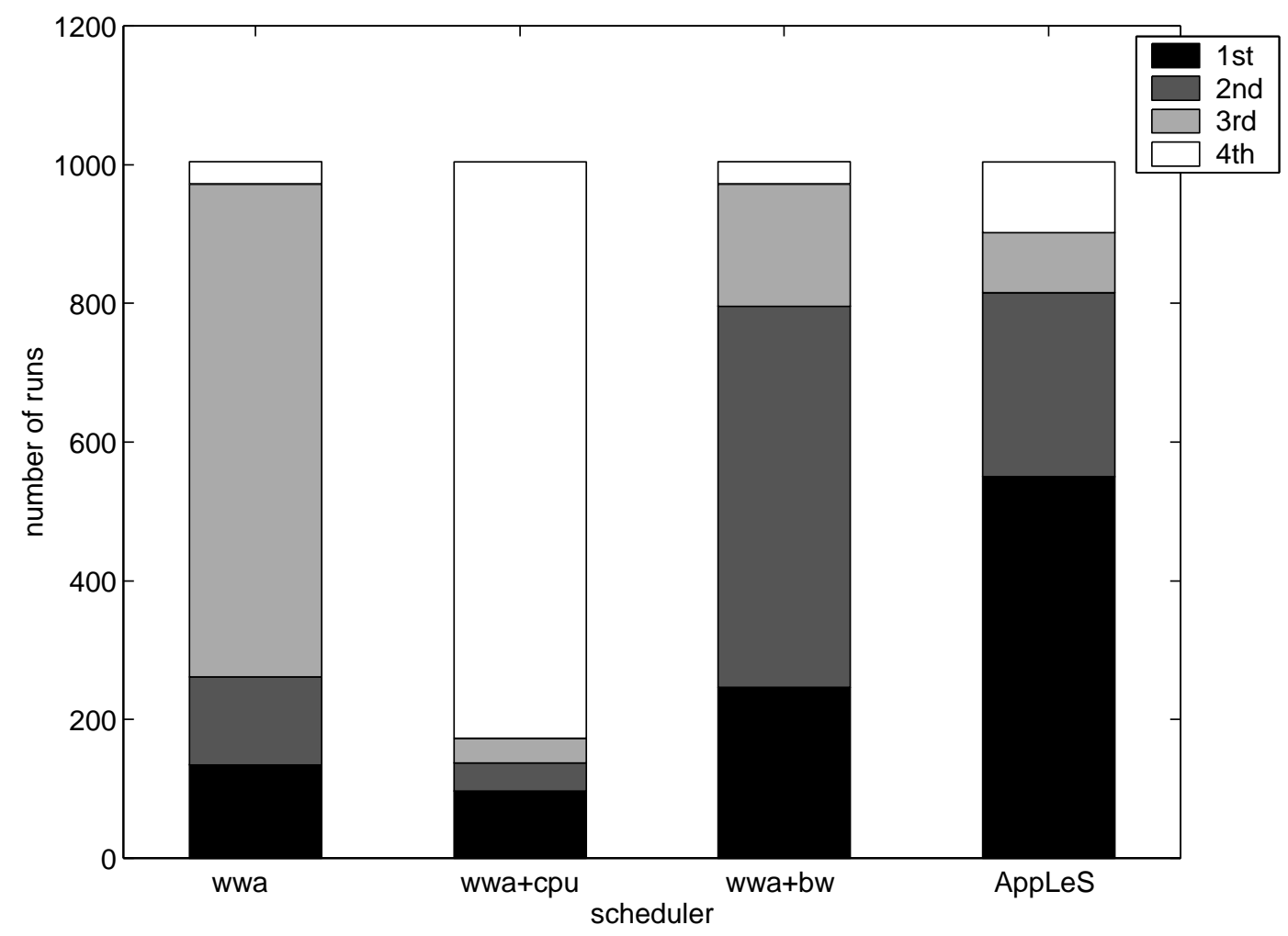

Fig. 13. Completely trace-driven simulations: scheduler ranking based on cumulative $\Delta$.

iment is displayed in Fig. 14; the range of $(f, r)$ pairs for the $E_{2}$ experiment is displayed in Fig. 15. For each pair, we show the percentage of the time it was feasible and optimal throughout the week. The percentage is visually depicted as variable-size $\times$ 's. Recall that our method filters out sub-optimal pairs (see Section 3.4). At this point we do not specify any model for the user pair selection criteria. For instance, if both pairs $(1,2)$ and $(2,1)$ are feasible for a given experiment, we plot both pairs in the figure and qualify both pairs as "optimal".

For both type of experiments the majority of feasible optimal pairs take two values: $(1,2)$ and $(2,1)$ for $E_{1}$ experiments; $(2,2)$ and $(3,1)$ for $E_{2}$ experiments. Note also that since the projections are larger for $E_{2}$ experiments, the scheduler opts for higher reduction factor values. These results indicate that in the NCMIR environment, different values for $f$ and $r$ should be used in order to best satisfy users' criteria. We are currently running experiments for many synthetic Grid environments modeled after real traces from actual Grid testbeds. Our preliminary results show that implementing tunability as part of on-line parallel tomography is critical over a wide range of computing environments.
In addition, in many cases the feasible optimal $(f, r)$ pairs take wider ranges of values than what we observe for the NCMIR Grid. We will report on those results in an upcoming research article.

In order to quantify the benefits of tunability as perceived by a user throughout time, we also performed the following experiment. We model a user who would choose a pair $(f, r)$ and then watch how that pair changes over time for back-to-back tomographic reconstructions. For these experiments, we assume a simple user model. We assumed that the user would always choose pairs that have the lowest $f$. We use the number of changes within a specified time period to measure the usefulness of tunability. For example, when the triple change frequency is low, we say that tunability is not useful. In other words, it is likely that a user could use the same configuration from run to run and not experience a significant drop in performance. Conversely, when the triple change frequency is high, we say that tunability is useful. We predict that a user running with the same configuration from run to run would experience significant performance drops and/or would under-utilize the resources.

We simulated tomographic reconstructions every 50 minutes throughout the week of traces (recall that a 


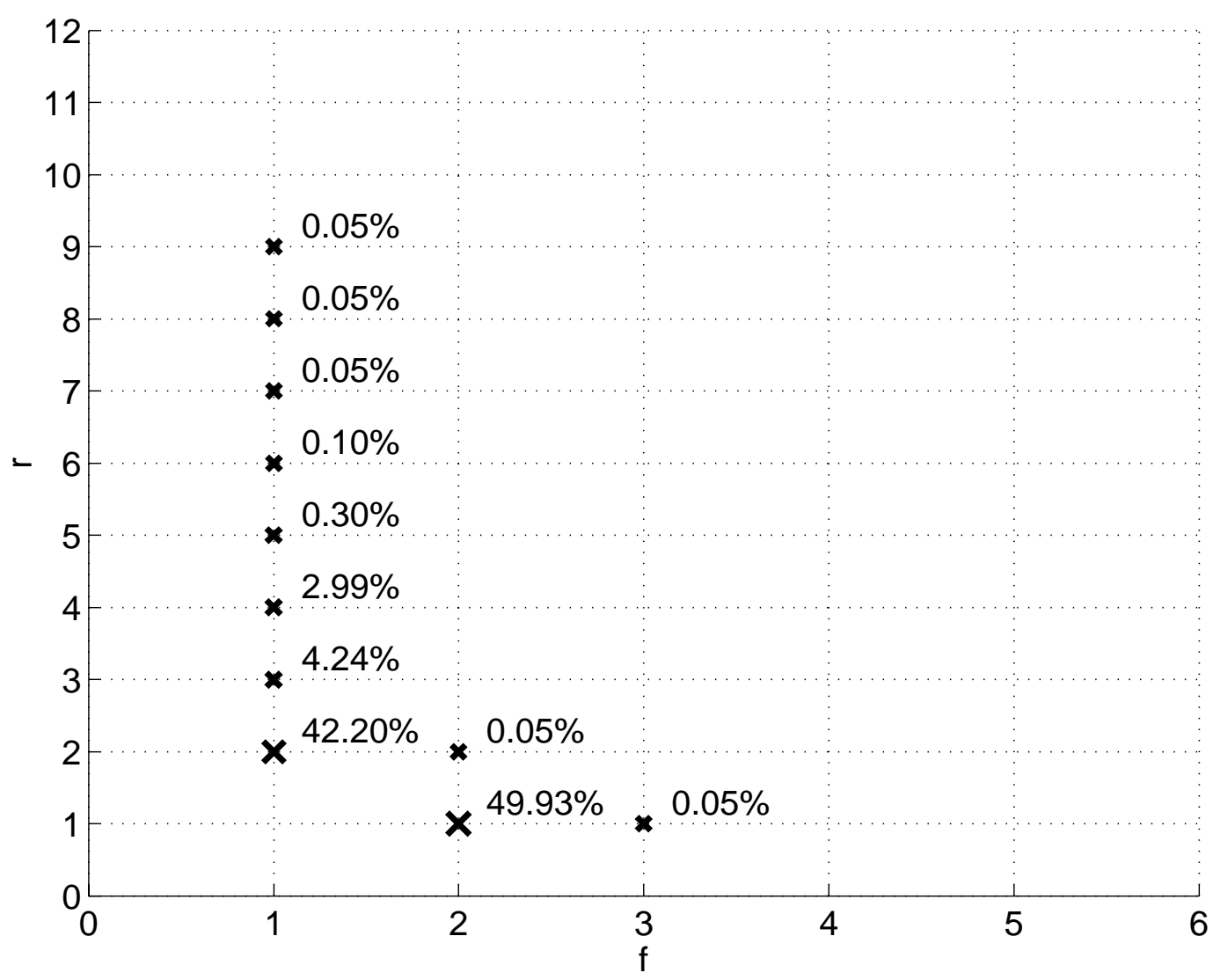

Fig. 14. $(f, r)$ pairs found for $(61,1024,1024,300)$ experiment.

Table 5

Evaluation of tunability using number of changes of the "best" tunable pair $(f, r)$ over a week on the NCMIR Grid

\begin{tabular}{cccc}
\hline Experiment type & $\begin{array}{c}\% \text { of } \\
\text { changes }\end{array}$ & $\begin{array}{c}\% \text { of } \\
\text { changes for } f\end{array}$ & $\begin{array}{c}\% \text { of } \\
\text { changes for } r\end{array}$ \\
\hline $1 k \times 1 k$ & $25.2 \%$ & $0.0 \%$ & $25.2 \%$ \\
$2 k \times 2 k$ & $25.1 \%$ & $22.9 \%$ & $19.2 \%$ \\
\hline
\end{tabular}

reconstruction takes 45 minutes). This corresponds to a user running 201 back-to-back reconstructions throughout the week. We performed simulations for $1 k \times 1 k$ and $2 k \times 2 k$ experiments, totaling 402 application simulations. For each reconstruction, we emulated the user and chose the "best" $(f, r)$ pair while monitoring changes of that pair from one experiment to the next.

First, we illustrate the usefulness of tunability by example. Figure 16 shows some of the pairs found on May 21, 2001. Suppose, the user starts a reconstruction at 8:00 a.m. using the pair $(3,1)$. If the user used the same configuration pair throughout the day, the user would not have been able to take advantage of the better configuration pairs available at 8:50 a.m. and 10:40 a.m. Conversely, the user would have observed very bad performance at 9:50 a.m. because there would not have been enough resources available to support the configuration pair $(3,1)$.

In total, we found that the $(f, r)$ pair changed 51 times out of the 201 reconstructions for the $E_{1}$ experiments. All changes were caused by the tuning of $r$. For the $E_{2}$ experiments, pairs changed 50 times out of the 201 reconstructions. In these 50 changes, 48 involved tuning of $r$, and 38 involved tuning of $f$. These results are summarized in Table 5 using percentages. In about $25 \%$ of the cases, is was a good choice to tune the application configuration (for both the $1 k \times 1 k$ and $2 k \times 2 k$ datasets) rather than using the previous configuration.

\section{Related work}

On-line parallel tomography has also been addressed as part of the Computed Microtomography (CMT) project $[40,41]$. Projections are collected from the Advanced Photon Source (APS) at Argonne National Lab- 


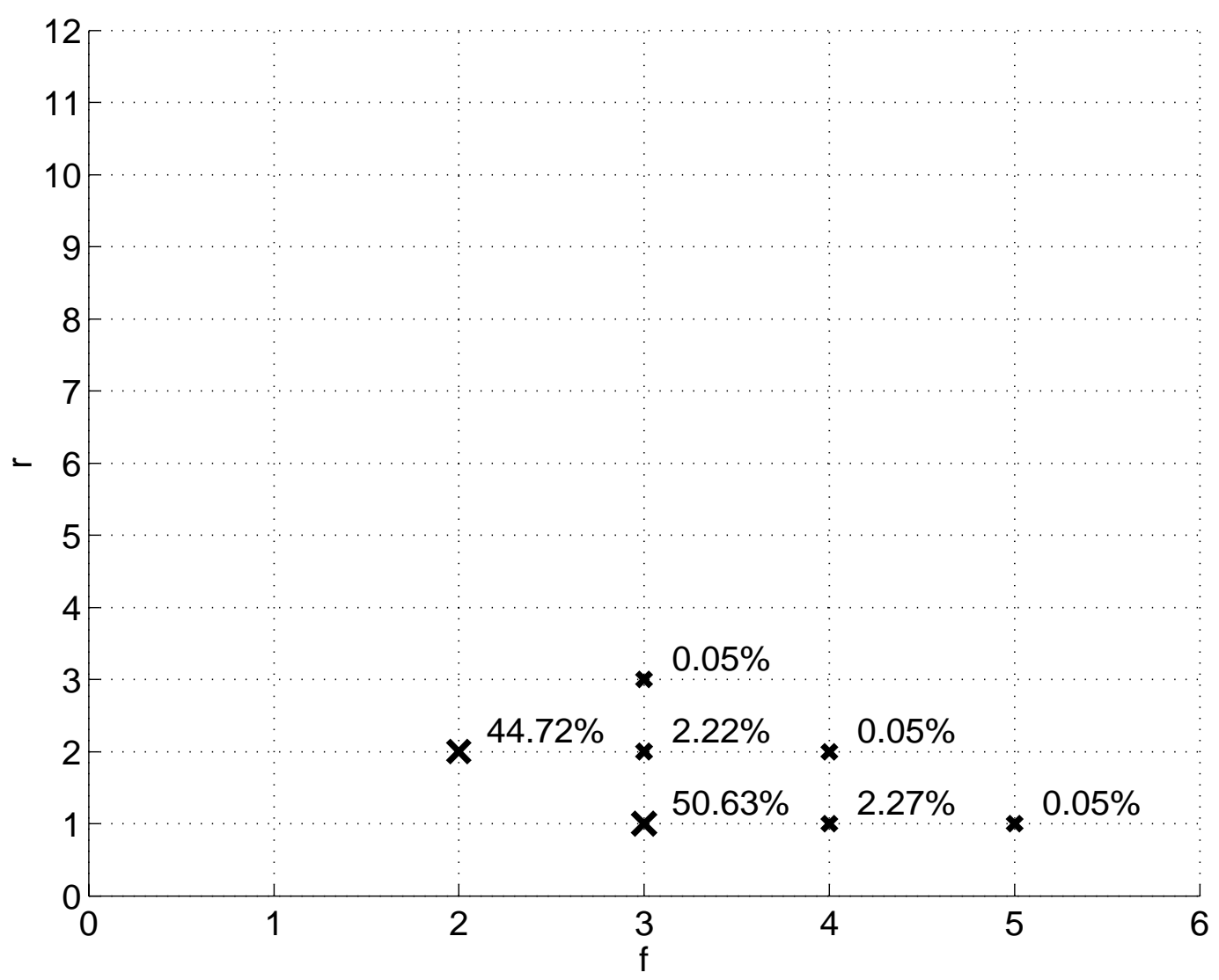

Fig. 15. $(f, r)$ pairs found for $(61,2048,2048,600)$ experiment.

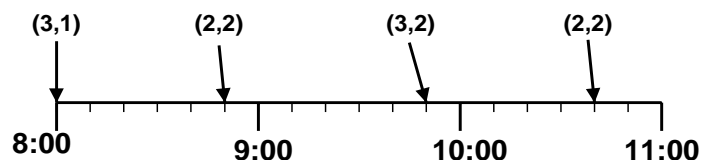

Fig. 16. Sample of configuration pairs chosen by our user model on May 21, 2001.

oratory, processed by an SGI Origin 2000, and visualized on an ImmersaDesk [42] or in a CAVE [43]. The CMT on-line parallel tomography code specifically targets high-speed networks and supercomputers and is a slightly extended version of the GTOMO code described in Section 2.2. The on-line parallel tomography implementation presented in this paper differs from CMT's in that it enables the R-weighted backprojection method to execute as an augmentable technique. Note that it would be straightforward to add the same extension to the CMT code in order to improve real-time execution. Second, our implementation enables on-line parallel tomography to execute across a more diverse set of resources (e.g., workstations, space-shared supercomputers, lower-capacity networks) through the use of application tunability and application-level scheduling.

Application tunability is a concept that has been applied in the MILAN project [7] and in [44]. In MILAN, tunability is used by the system scheduler to improve throughput. The system scheduler is referred to as the $Q o S$ arbitrator and is responsible for allocating processors to application tasks. Each application has a QoS agent which interacts with the QoS arbitrator to ensure that its execution requirements are being satisfied. The QoS agent is automatically generated from annotated code. Our work differs from MILAN's in that our objective is to use tunability to improve application performance rather than system performance. We provide a single AppLeS process which functions as both the application's QoS agent and QoS arbitrator. While MILAN provides a simpler API, it is currently unable to sufficiently capture the requirements of on-line parallel tomography because the QoS arbitrator does not schedule bandwidth on network links. Given the large amount of data transfer required for on-line parallel tomography, the ability to express bandwidth 
requirements is critical to achieving real-time execution performance.

The work presented in [44] also uses tunability to improve application performance. Two applications are presented and classified as prediction-based, best effort, real-time applications. Using predictions of application performance based on dynamic load predictions, the application is mapped to a set of resources. Our work differs from theirs in that predictions of application performance are model-based rather than historybased.

The concept of soft deadlines for computing on the Grid has been explored in the Nimrod/G project [45]. In Nimrod/G scheduling aims at achieving trade-offs between deadline requirements and resource cost. In this work, our contribution is that we perform trade-offs between resource availability and key characteristics of the application's output. In other words, we studied soft deadline scheduling in the context of tunable applications. In future work, we will add Nimrod/G's notion of resource cost to our current scheduling model (see Section 6).

Finally, the AppLeS described in this paper builds upon other previous AppLeS work [18-20,4] in its strategies for resource selection and work allocation. These AppLeS have focused on improving the performance of applications with fixed configurations. The AppLeS described herein distinguishes itself from these schedulers in its ability to improve the performance of an application (with multiple configurations) by exploiting its tunability.

\section{Conclusion}

We have extended our previous work on off-line parallel tomography [4] to address the on-line scenario. We have modeled our application as a tunable application, allowing users to express trade-offs between tomogram resolution and refresh rates. Our scheduling strategy uses dynamic CPU and network bandwidth availability information to perform resource selection. We have identified scheduling/tuning in terms of multiple constrained optimization problems. Simulation results showed that our scheduler chooses appropriate work allocations because it takes into account dynamic bandwidth information. Finally, we demonstrate the importance of tunability in a computing environment such as the one at NCMIR.

In future work, we will explore the notion of cost for resource usage. Several supercomputer centers reg- ulate resource access with allocations and tunability can then be expressed as a triple $(f, r, c o s t)$ where cost is the allocation units the user is willing to spend. The same optimization techniques as described in Section 3.4 apply. The notion of cost and soft deadline has been explored in [45]. Our contribution is that we allow for tunability in terms of key parameters of the target applications. Also, we are currently running simulations for synthetic computing environments and a future paper will present an evaluation of our scheduling/tuning strategy for environments with various topologies and resource availabilities. The implementation of on-line parallel tomography described in this paper will be put into production mode at NCMIR. We expect this will allow NCMIR users to acquire higher quality data from their electron microscope and will allow for more efficient use of this scarce resource.

Finally, our work on on-line tomography is applicable to a large class of applications. Our methodology provides a general framework for scheduling tunable applications with soft deadline requirements in Grid environments.

\section{Acknowledgements}

The authors would like to thank the reviewers, members of the Grid Computing Laboratory, and our colleagues at NCMIR and ISI for their insightful comments. Particularly, we are grateful to Holly Dail for reviewing earlier drafts of this paper. We would also like to thank Phil Papadopoulos for use of the Meteor cluster at SDSC and David Hutches for use of the Active Web cluster at UCSD.

This research was supported by NSF grants ACI9701333 and ACS-9619020. Equipment used in this research was supported in part by the UCSD Active Web Project, NSF Research Infrastructure Grant Number 9802219 .

\section{References}

[1] A. C. Kak and M. Slaney, Principles of Computerized Tomography Imaging, IEEE Press, 1998.

[2] G.A. Perkins, C.W. Renken, S.J. Young, S.P. Lamont, M.E. Martone, S. Lindsey, T.G Frey and M.H. Ellisman, Electron tomography of large multicomponent biological structures. $J$. Struct. Biol. 120 (1997), 219-227.

[3] J. Frank and M. Radermacher, Three-Dimensional Reconstruction of Nonperiodic Macromolecular Assemblies from Electron Micrographs, in: Advanced Techniques in Biological Electron Microscopy III, J.K. Koehler, ed., Springer-Verlag, 1986. 
[4] Shava Smallen, Walfredo Cirne, Jaime Frey, Francine Berman, Rich Wolski, Mei-Hui Su, Carl Kesselman, Steve Young and Mark Ellisman, Combining Workstations and Supercomputers to Support Grid Applications: The Parallel Tomography Experience, Proceedings of the 9th Heterogenous Computing Workshop, May 2000.

[5] Ian Foster and Carl Kesselman, eds, The Grid: Blueprint for a New Computing Infrastructure, Morgan Kaufmann Publishers, Inc., San Francisco, USA, 1999.

[6] I. Foster, C. Kesselman and S. Tuecke, The Anatomy of the Grid: Enabling Scalable Virtual Organizations, Intl. J. Supercomputer Applications (2001), in press.

[7] Fangzhe Chang, Vijay Karamcheti and Zvi Kedem, Exploiting Application Tunability for Efficient, Predictable Resource Management in Parallel and Distributed Systems, Journal of Parallel and Distributed Computing 60 (2000), 1420-1445.

[8] Francine Berman, Richard Wolski, Silvia Figueira, Jennifer Schopf and Gary Shao, Application Level Scheduling on Distributed Heterogeneous Networks, Proceedings of Supercomputing 1996, 1996.

[9] F. Berman and R. Wolski, The AppLeS Project: A Status Report, Proc. of the 8th NEC Research Symposium, Berlin, Germany, May 1997.

[10] M. Radermacher, Three-dimensional reconstruction of single particles from random and nonrandom tilt series. J. Electron Microsc. Tech. 9 (1988), 359-394.

[11] R. Gordon, R. Bender and G.T. Herman, Algebraic Reconstruction Techniques (ART) for Three-dimensional Electron Microscopy and X-ray Photography. J. Theoret. Biol. 29 (1970), 471-481.

[12] P. Gilbert, Iterative Methods for the Three-dimensional Reconstruction of an Object from Projections, J. Theoret. Biol. 36 (1972), 105-117.

[13] Ian Foster and Carl Kesselman, The Globus Project: A Status Report, Proc. IPPS/SPDP '98 Heterogeneous Computing Workshop, 1998.

[14] A. Grimshaw, A. Ferrari, F.C. Knabe and M. Humphrey, WideArea Computing: Resource Sharing on a Large Scale, IEEE Computer 32(5) (May, 1999).

[15] M.J. Litzkow, M. Livny and M.W. Mutka, Condor - A Hunter of Idle Workstations, Proc. of the 8th Int'l Conf. on Distributed Computing Systems, 1988, pp. 104-111.

[16] Henri Casanova and Jack Dongarra, NetSolve: A Network Server for Solving Computational Science Problems, The International Journal of Supercomputing Applications and High Performance Computing, 1996.

[17] S. Sekiguchi, M. Sato, H. Nakada, S. Matsuoka and U. Nagashima, Ninf: Network based Information Library for Globally High Performance Computing, Proc. of Parallel Object-Oriented Methods and Applications (POOMA), February 1996, pp. 39-48.

[18] Alan Su, Francine Berman, Richard Wolski and Michelle Mills Strout, Using AppLeS to Schedule Simple SARA on the Computational Grid, International Journal of High Performance Computing Applications 13(3) (1999), 253-262.

[19] Neil Spring and Rich Wolski, Application Level Scheduling of Gene Sequence Comparison on Metacomputers, 12th ACM International Conference on Supercomputing, July, 1998.

[20] Holly Dail, Graziano Obertelli, Francine Berman, Rich Wolski and Andrew Grimshaw, Application-Aware Scheduling of a Magnetohydrodynamics Application in the Legion Metasystem, Proceedings of the 9th Heterogenous Computing Workshop, May, 2000.
[21] T. Hagerup, Allocating Independent Tasks to Parallel Processors: An Experimental Study, Journal of Parallel and Distributed Computing 47 (1997), 185-197.

[22] Gabriel E. Soto, Stephen J. Young, Maryann E. Martone, Thomas J. Deerinck, Stephan Lamont, Bridget O. Carragher, Kiyoshi Hamma and Mark H. Ellisman, Serial section electron tomography: A method for three-dimensional reconstruction of large structures, Neuroimage 1 (1994), 230-243.

[23] Reinhard Klette and Piero Zamperoni, Handbook of Image Processing Operators, (chapter 4), John Wiley and Sons, Ltd., 1996, pp. 120-125.

[24] Jane W.S. Liu, Real-Time Systems, (chapter 2), Prentice-Hall, Inc., 2000, pp. 26-33.

[25] Stefan D. Bruda and Selim G. Akl, Real-Time Computation: A Formal Definition and its Applications, Technical Report 435, Queen's University, 2000.

[26] Rich Wolski, Neil T. Spring and Jim Hayes, The Network Weather Service: A Distributed Resource Performance Forecasting Service for Metacomputing, The Journal of Future Generation Computing Systems (1999).

[27] Maui Scheduler webpage at http://www.mhpcc.edu/maui.

[28] David E. Culler and Jaswinder Pal Singh, Parallel Computer Architecture, (chapter 1), Morgan Kaufmann Publishers, Inc., 1999, pp. 60-61.

[29] Andrew S. Tanenbaum, Computer Networks, (chapter 1), Prentice Hall, Inc., Third edition, 1996, pp. 8.

[30] Radia Perlman, Interconnections, (chapter 2), Addison Wesley Longman, Inc., second edition, 2000, pp. 19.

[31] Gary Shao, Fran Berman and Rich Wolski, Using Effective Network Views to Promote Distributed Application Performance, Proceedings of the 1999 International Conference on Parallel and Distributed Processing Techniques and Applications, 1999.

[32] Linear Programming FAQ webpage at http://www-unix.mcs. anl.gov/otc/Guide/faq/linear-programming-faq.html.

[33] Nonlinear Programming FAQ webpage at http://www-unix. mcs.anl.gov/otc/Guide/faq/nonlinear-programming-faq.html.

[34] lp_solve FTP site at ftp://ftp.es.ele.tue.nl/pub/lp solve.

[35] Dimitri P. Bertsekas, Nonlinear Programming, (chapter 1), Athena Scientific, 1999, pp. 2.

[36] Henri Casanova, Arnaud Legrand, Dmitrii Zagorodnov and Francine Berman, Heuristics for Scheduling Parameter Sweep applications in Grid Environments, Proceedings of the 9th Heterogenous Computing Workshop, May, 2000.

[37] Henri Casanova, Simgrid: A Toolkit for the Simulation of Application Scheduling, Proceedings of the IEEE/ACM International Symposium on Cluster Computing and the Grid, May, 2001.

[38] H. Casanova, G. Obertelli, F. Berman and R. Wolski, The AppLeS Parameter Sweep Template: User-Level Middleware for the Grid. Proceedings of SuperComputing'00, November, 2000.

[39] Atsuko Takefusa, Satoshi Matsuoka, Hidemoto Nakada, Kento Aida and Umpei Nagashima, Overview of a Performance Evaluation System for Global Computing Scheduling Algorithms, Proceedings of the 8th IEEE International Symposium on High Performance Distributed Computing (HPDC), August, 1999, pp. 97-104.

[40] G. von Laszewski, M.-H. Su, J. Insley, I. Foster, J. Bresnahan, C. Kesselman, M. Thiebaux, M. Rivers, S. Wang, B. Tieman and I. McNulty, Real-Time Analysis, Visualization and Steering of Tomography Experiments at Photon Sources, Ninth SIAM Conference on Parallel Processing for Scientific Computing, Apr, 1999. 
[41] Y. Wang, F. De Carlo, I. Foster, J. Insley, C. Kesselman, O. Lane, G. von Laszewski, D. Mancini, I. McNulty, M.-H. Su and B. Tieman, A quasi-realtime xray microtomography system at the Advanced Photon Source, Proceedings of SPIE 3772 (1999).

[42] M. Czernuszenko, D. Pape, D. Sandin, T. DeFanti, G. Dawe and M. Brown, The ImmersaDesk and Infinity Wall Projection-Based Virtual Reality Displays, Computer Graphics 31(2) (1997), 46-49.

[43] C. Cruz-Neira, D. Sandin and T. DeFanti, Surround-Screen Projection-Based Virtual Reality: The Design and Implemen- tation of the CAVE, ACM Computer Graphics 27(2) (July, 1993), 135-142.

[44] Peter A. Dinda, Bruce Lowekamp, Loukas Kallivokas and David R. O'Hallaron, The Case for Prediction-based Besteffort Real-time Systems, Technical Report CMU-CS-98-174, Carnegie Mellon University, 1999.

[45] D. Abramson, J. Giddy and L. Kotler, High Performance Parametric Modeling with Nimrod/G: Killer Application for the Global Grid? Proceedings of the International Parallel and Distributed Processing Symposium (IPDPS), Cancun, Mexico, May, 2000, pp. 520-528. 

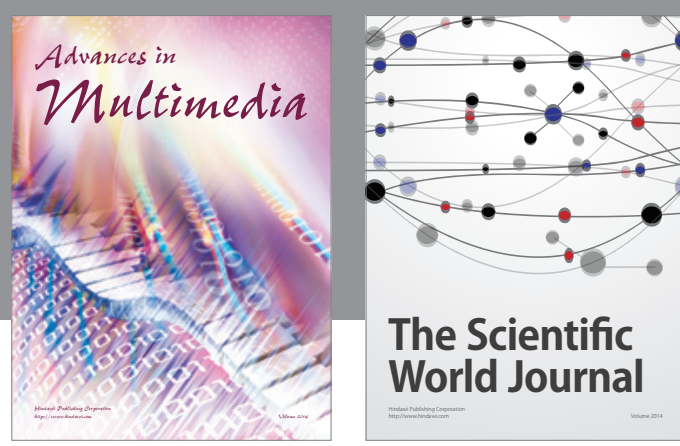

The Scientific World Journal
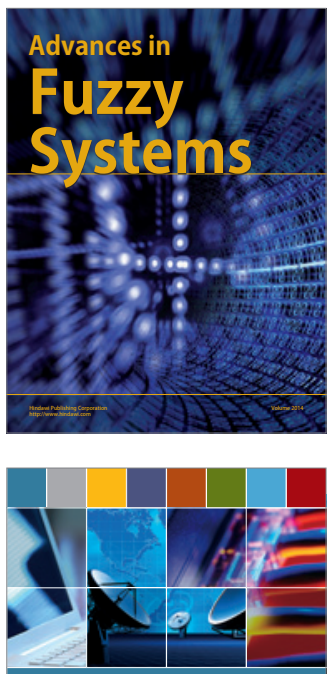

Computer Networks and Communications
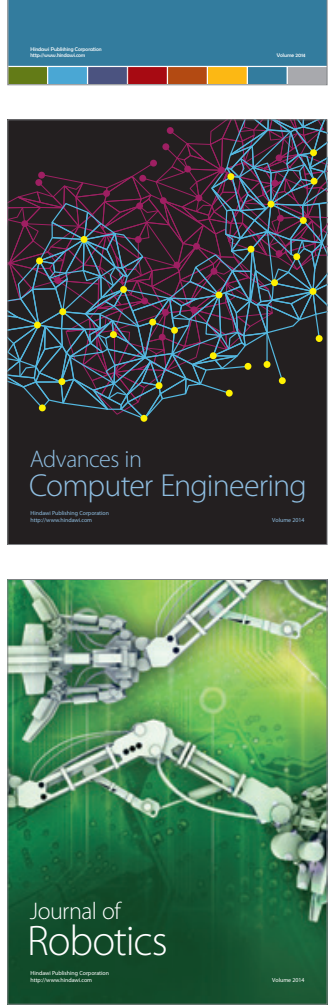
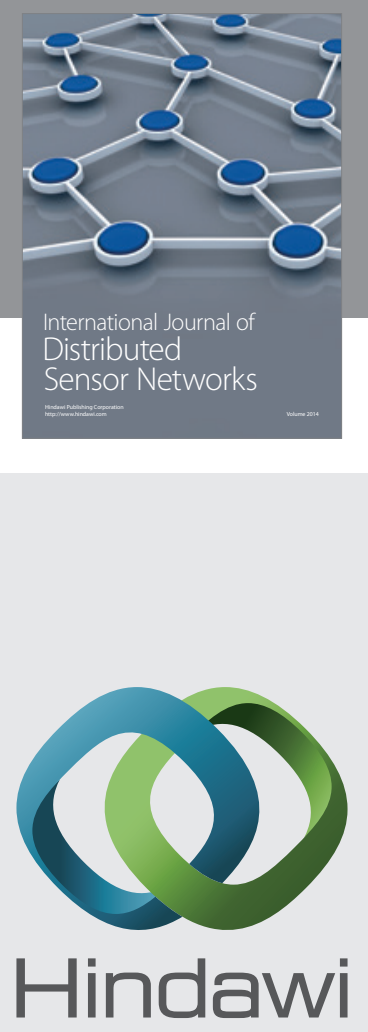

Submit your manuscripts at

http://www.hindawi.com
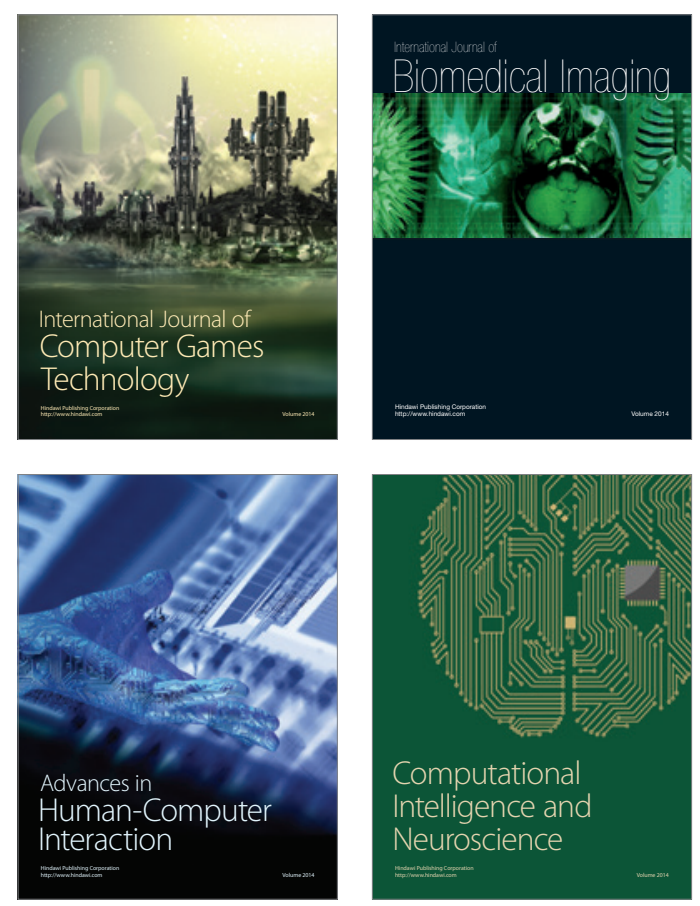
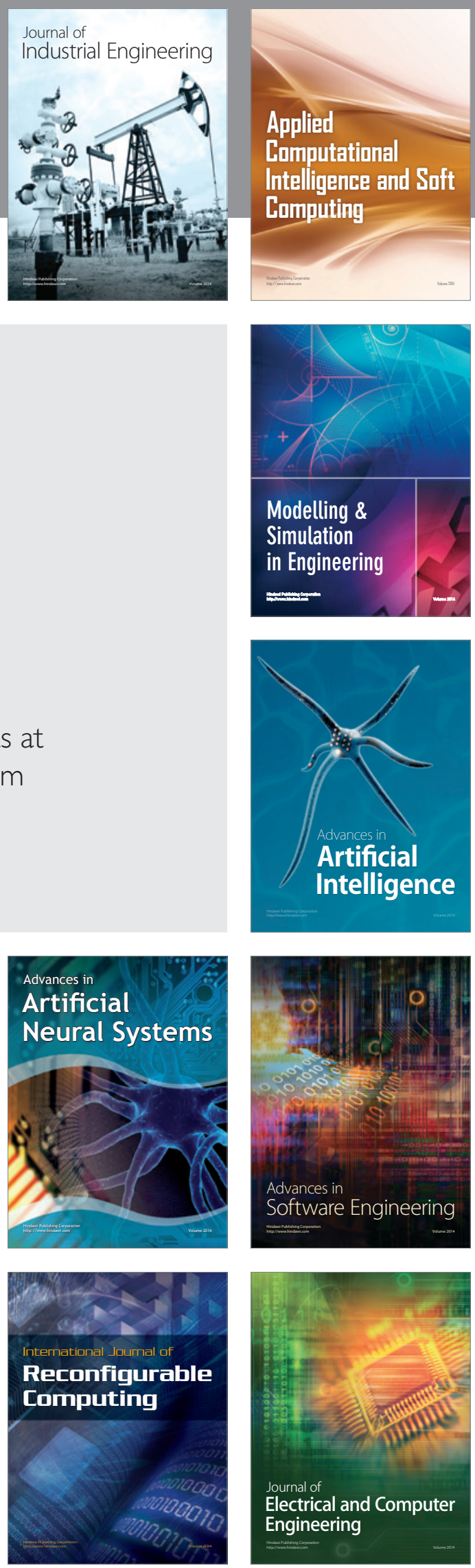